\title{
Aplicación gecotoxic para predicción de riesgo ambiental: caso estudio sobre mortandad de peces en la bahía interior del lago Titicaca-Puno, Perú
}

Gecotoxic application for predicting risk environmental: case study on mortality in fish inner bay of Lake Titicaca-Puno, Peru

\begin{tabular}{l|l|l} 
Recibido: agosto 10 de 2015 & Revisado: setiembre14 de 2015 Aceptado: octubre 12 de 2015
\end{tabular}

George Argota Pérez ${ }^{\mathrm{I}}$

1 Dirección General. Centro de Investigaciones Avanzadas y Formación Superior en Educación, Salud y Medio Ambiente "AMTAWI".

george.argota@gmail.com

\begin{abstract}
The aim of this study was to apply the GECOTOXIC program for predicting environmental risk of fish kills in the inner bay of Lake Titicaca, Puno-Peru. To do the study was conducted in March 2013, where the test area corresponded to the area of Chimu. Random probability sampling type previously made, while the research method was quantitative empirical observation and measurement of both. The variables analyzed were for the identification of major pollution sources, physico-chemical, meteorological variables, as well as biometrics and histopathology in fish Orestias agassii "carachi gray" Orestias luteus "yellow carachi" Odontesthes bonariensis "silverside" disparate Trichomycterus " mauri ". With all these analyzes subsequently were introduced to GECOTOXIC program, which is multifunctional type on real data. Each of the program's menus threw a kind of results where the effect of interaction, he finally said predicting environmental risk was tall.
\end{abstract}

Keywords: gecotoxic, predicting environmental risk, mortality, fish, inner bay, Lake Titicaca, Puno

\section{RESUMEN}

El objetivo de este trabajo fue aplicar el programa GECOTOXIC para la predicción de riesgo ambiental sobre la mortandad de peces en la bahía interior del lago Titicaca, Puno-Perú. Para ello, el estudio se efectuó en marzo del 2013, donde la zona de análisis correspondió al área de Chimú. Se realizó, previamente, un muestreo de tipo probabilístico aleatorio, mientras que el método cuantitativo de investigación fue empírico tanto de observación como de medición. Las variables analizadas correspondieron a la identificación de principales fuentes contaminantes, parámetros físico-químicos, variables meteorológicas, así como biometría e histopatología en los peces Orestias agassii "carachi gris", Orestias luteus "carachi amarillo", Odontesthes bonariensis "pejerrey" Trichomycterus dispar "mauri". Con todos estos análisis, posteriormente, fueron introducidos al programa GECOTOXIC, que es de tipo multifuncional sobre datos reales. Cada uno de los menús del programa arrojó un tipo de resultados por efecto de interacción. Finalmente, se indicó que la predicción de riesgo ambiental fue de tipo alto.

Palabras clave: gecotoxic, predicción de riesgo ambiental, mortandad, peces, bahía interior, lago Titicaca, Puno 


\section{Introducción}

Es importante destacar, que en el pasado siglo XX y lo que transcurre en este siglo XXI, es más acuciante la contaminación ambiental como una de las situaciones ambientales globales (Samantray, Mishra, Panda \& Rout, 2009). En tal sentido, si bien es cierto que los gobiernos están adoptando medidas con carácter normativo, así como determinadas políticas para minimizar los riesgos ambientales y la salud pública, puede mencionarse que todavía faltan esfuerzos relacionados con la gestión científica para la búsqueda de mecanismos reguladores más eficientes, pues si bien es cierto que la evaluación de riesgo es una herramienta científica de vital aplicación (Vanrolleghem, Young, Morris, Gandolfi \& Feijtel, 2001), aún se necesita la incorporación de variables en forma más precisa e integrar conjuntamente con acciones simultáneas para considerar la estimación de efectos e impactos por exposición a cargas contaminantes con la mayor precisión y exactitud posible.

La evaluación de riesgo no necesariamente requiere la aplicación de técnicas sofisticadas o la recolección excesiva de datos. Se pueden obtener resultados prácticos y razonables utilizando información mínima disponible sobre la contaminación y sobre la población expuesta a ella (World Bank, 1998; Lee, 2009).

Según Morgan \& Henrion (1990), las evaluaciones de riesgo proporcionan un valor simple como una estimación conservadora del riesgo, mientras que hoy se acepta, por lo general, que en la caracterización del riesgo se requiere un mayor entendimiento de los métodos de estimación y de la incertidumbre involucrada en la estimación.

Carpenter (1995) señala que la evaluación de riesgo ambiental (ERA) sigue las reglas de la teoría de la probabilidad mediante una expresión de todos los posibles valores de cada parámetro analizado. Management Programme for the Gordon Development (MPGD, 2004) indica que la ERA es un proceso que evalúa la probabilidad y consecuencia de un impacto adverso que ocurre como resultado de la exposición a uno o más estresores.

Por su parte, Der Oost, Beyer \& Vermeulen (2003) argumentan que deberían analizarse biomarcadores como proteínas de estrés, metalotioneínas, parámetros hematológicos, inmunológicos, genotóxicos, endocrinos, etc., así como marcadores de bioacumulación referidos a xenobióticos, empleándolos en la ERA que, definitivamente, es un procedimiento por el cual de forma probable o real, un efecto adverso de un contaminante y otras actividades antropogénicas en el ecosistema y sus componentes son estimados con un grado de incertidumbre y que realmente son usados en cualquier metodología científica.

En cuanto a esta incertidumbre, en la evaluación de riesgo puede originarse por distintas causas como pueden ser la falta de información, diferencias en el nivel de la evidencia, simplificaciones o suposiciones realizadas para hacer factible el análisis, siendo importante distinguir entre incertidumbre $y$ variabilidad, debido a que ambas pueden ocasionar incertidumbres en los resultados de la evaluación de riesgo (Darbra, Eljarrat \& Barceló, 2008). La incertidumbre se genera por el conocimiento incompleto del valor verdadero de un parámetro y se origina en la necesidad de establecer inferencias para pequeñas muestras sobre los procesos que tienen un cierto grado de aleatoriedad (Evans, 2002).

Particularmente, las evaluaciones de riesgo en los ecosistemas, no siempre cuentan con el uso de organismos naturales, por lo que, en algunos casos, han sido aplicados índices basados en modelaciones. Determinados modelos cuantitativos expresan cambios 
en condiciones iniciales, valores de parámetros y estructura de relaciones funcionales que a menudo, ejercen impactos importantes sobre los resultados del modelo. Dambacher \& Ramos (2007) señalan que los modelos cuantitativos solo pueden llevarse a cabo con un nivel alto de conocimiento del sistema de interés o focal.

Argota \& Iannacone (2014) refirieron que aún faltan estudios metodológicos y/o programas computacionales que puedan referirse no solo a la caracterización de la entidad contaminante, sino además al análisis simultáneo entre las variables definidas como residuales tributados, efectos ecotoxicológicos y la significación del sistema de tratamiento ambiental aplicado, donde finalmente puedan compararse las evaluaciones de riesgo realizadas con los efectos en los organismos desarrollados naturalmente.

El objetivo de este trabajo fue aplicar el programa GECOTOXIC para la predicción de riesgo ambiental sobre la mortandad de peces en la bahía interior del lago Titicaca, Puno-Perú.

\section{Método}

- Objeto de investigación y período de estudio

El estudio se realizó en marzo de 2013. La zona de análisis correspondió al área de Chimú, la cual se encuentra próxima a la bahía interior del Lago Titicaca en la ciudad de Puno, Perú (Programa de las Naciones Unidas para el Desarrollo, 2003).

- Población y muestra

Del total de fuentes contaminantes tributarias a la bahía interior, fueron identificadas como principales responsables directas de la zona de estudio, las pozas de tratamientos las cuales fueron clasificadas como no funcionales.
En relación con los parámetros de calidad del agua, fueron determinados in-situ mediante el medidor multiparamétrico Hanna HI 9828. Se midió la temperatura del agua, oxígeno disuelto y dióxido de carbono, que fueron reportados por la Universidad Nacional del Altiplano en Puno. En el caso de la demanda química de oxígeno, nitritos, nitratos, nitrógeno amoniacal, fosfato, aceites y grasas como sólidos totales que fueron determinados por el Laboratorio Continental con sede en Puno (Instituto del Mar del Perú, 2013).

Asimismo, fue considerada la temperatura del aire, vientos y radiación solar como variables meteorológicas que se registraron en Estación Meteorológica de las islas flotantes de los Uros en Puno (IMARPE, 2013).

Finalmente, se realizó en las especies Orestias agassii "carachi gris", Orestias luteus "carachi amarillo", Odontesthes bonariensis "pejerrey" y Trichomycterus dispar "mauri", análisis de biomasa e histopatología en branquias.

- Análisis estadístico

Fue aplicada la metodología propuesta por Argota \& Iannacone (2014a), la cual predice el riesgo ecotoxicológico por exposición a efectos contaminantes en efluentes ambientales y ecosistemas acuáticos.

\section{Resultados}

En la Tabla 1, pueden mostrarse los principales parámetros físico-químicos de calidad ambiental de agua que justificaron el mayor peso como la variabilidad dentro del ecosistema bahía interior de Puno durante el muestreo que generó la mortandad de las especies de peces. 
Tabla 1

Parámetros físico-químicos de calidad ambiental de agua

\begin{tabular}{|l|l|c|}
\hline \multicolumn{1}{|c|}{ Laboratorio } & \multicolumn{1}{|c|}{ Parámetros } & Promedio \\
\hline \multirow{2}{*}{$\begin{array}{l}\text { Facultad de Biología y Mega- } \\
\text { laboratorio. Universidad Nacional } \\
\text { del Altiplano }\end{array}$} & Temperatura & 17.8 \\
\cline { 2 - 3 } $\begin{array}{l}\text { Laboratorio Continental en Puno, } \\
\text { Instituto del Mar del Perú }\end{array}$ & Oxeno disuelto & 0.88 \\
\cline { 2 - 3 } & DQÓxido de carbono & 18.4 \\
\cline { 2 - 3 } & $\mathrm{DBO}_{5}$ & 26.31 \\
\cline { 2 - 3 } & $\mathrm{NO}_{2}$ & 10.12 \\
\cline { 2 - 3 } & $\mathrm{NO}_{3}$ & 0.037 \\
\cline { 2 - 3 } & $\mathrm{NH}_{3}-\mathrm{N}$ & 0.044 \\
\cline { 2 - 3 } & $\mathrm{PO}_{4}$ & 1.79 \\
\cline { 2 - 3 } & aceites y grasas & 2.20 \\
\cline { 2 - 3 } & $\mathrm{ST}$ & 0.9 \\
\hline
\end{tabular}

En la Figura 1, se muestra el comportamiento de variables meteorológicas registra- do por la Estación Meteorológica de la isla flotantes de los uros en Puno.
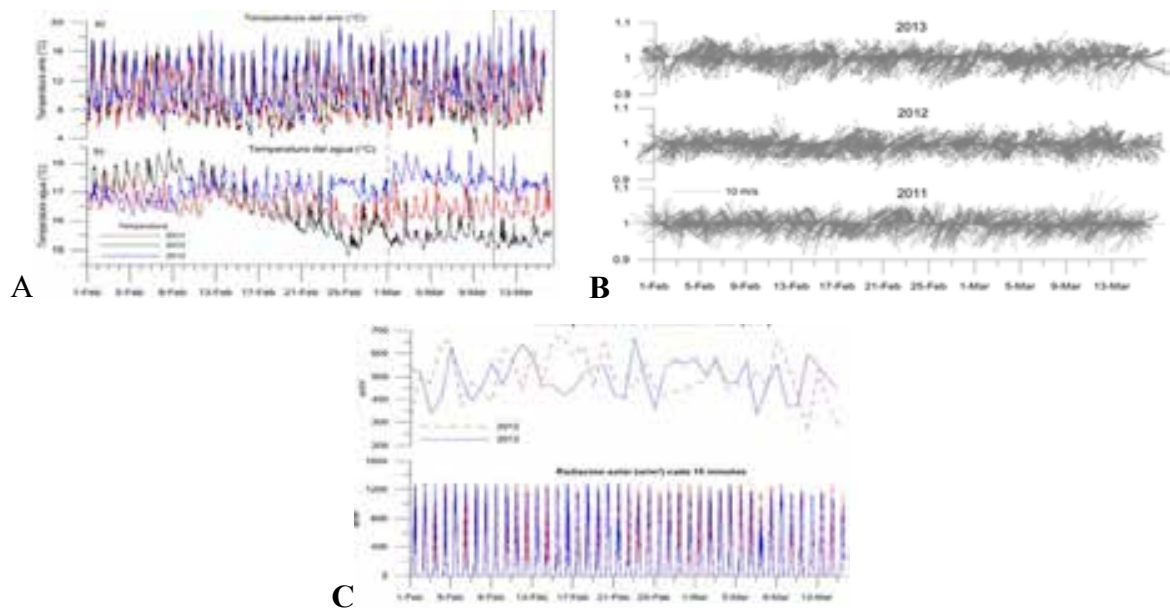

Figura 1. Comportamiento de la temperatura del aire (A), vientos (B) y radiación solar (C).

En la Tabla 2, pueden mostrarse la biomasa y el número de Orestias agassii "carachi gris", Orestias luteus "carachi amarillo",
Odontesthes bonariensis "pejerrey" y Trichomycterus dispar "mauri", estimados por mortandad.

Tabla 2

Biomasa y número de ejemplares estimados por mortandad

\begin{tabular}{|c|c|c|}
\hline Especie & Biomasa $(\mathrm{Kg})$ & $\mathbf{N}^{\circ}$ \\
\hline $\begin{array}{l}\text { Orestias agassii "carachi gris" } \\
\text { Orestias luteus "carachi amarillo" } \\
\text { Odontesthes bonariensis "pejerrey" } \\
\text { Trichomycterus dispar "mauri" }\end{array}$ & $\begin{array}{r}1226,2 \\
702,0 \\
271,4 \\
140,4\end{array}$ & $\begin{array}{r}65000 \\
44000 \\
6500 \\
3900\end{array}$ \\
\hline Total & 2340,0 & 119400 \\
\hline
\end{tabular}

Nota: Laboratorio Continental del IMARPE, Puno (2013).

En la Tabla 3, se muestra el análisis estadístico de los datos, donde según la prueba de la menor diferencia estadística de Fisher se en- contró, variabilidad entre las especies existiendo, diferencias estadísticamente significativas $(\mathrm{p} \leq 0.05)$ dada la pérdida por mortandad. 
Tabla 3

Análisis estadistico de los datos

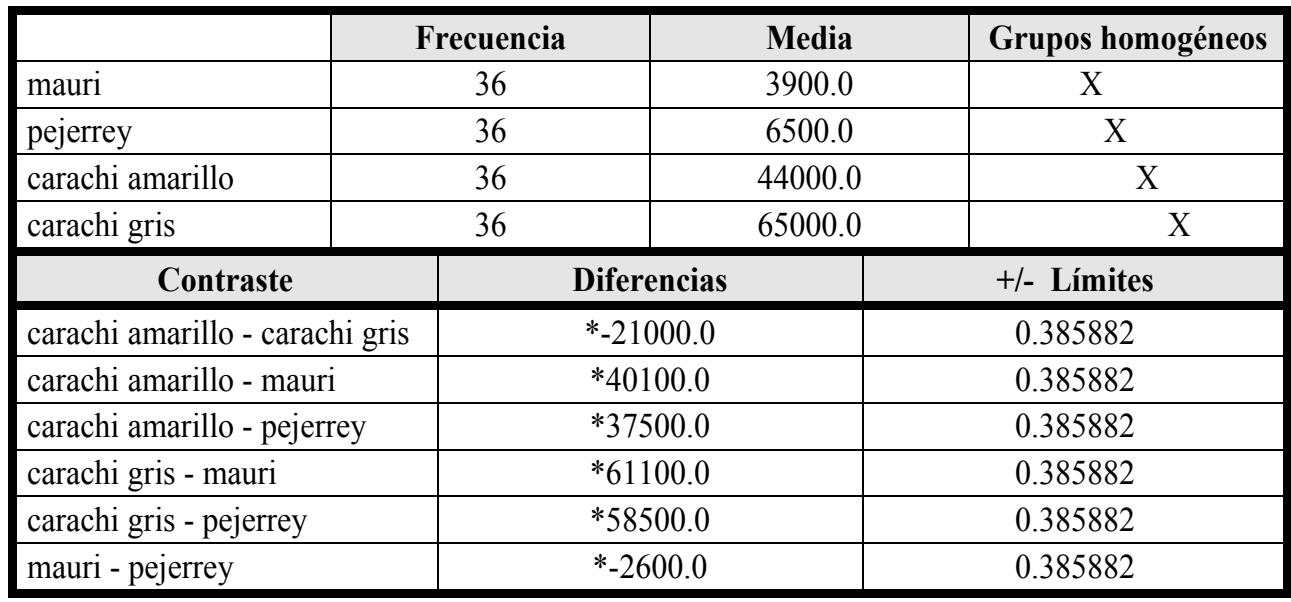

* indica una diferencia significativa

En la Tabla 4, pueden mostrase el rango y valor promedio de Orestias agassii "carachi gris", Orestias luteus "carachi amarillo",

Tabla 4

Rango de tallas y valor promedio por especies (cm)

\begin{tabular}{|l|c|c|}
\hline \multicolumn{1}{|c|}{ Especie } & Rango (cm) & Media (cm) \\
\hline Orestias agassii "carachi gris" & $7,5-16,0$ & 10,0 \\
Orestias luteus "carachi amarillo" & $6,5-14,5$ & 10,4 \\
Odontesthes bonariensis "pejerrey" & $6,5-30,5$ & 15,1 \\
Trichomycterus dispar "mauri" & $14,0-15,5$ & 14,8 \\
\hline
\end{tabular}

Nota: Laboratorio Continental del IMARPE, Puno (2013).

En la Figura 2, se muestra el daño histopatológico encontrado en branquias de las especies Orestias agassii "carachi gris", Orestias luteus "carachi amarillo", Odontesthes bonariensis "pejerrey" y Trichomycterus dispar "mauri" (izquierda) dada la mortandad,
Odontesthes bonariensis "pejerrey" y Trichomycterus dispar "mauri" por mortandad.

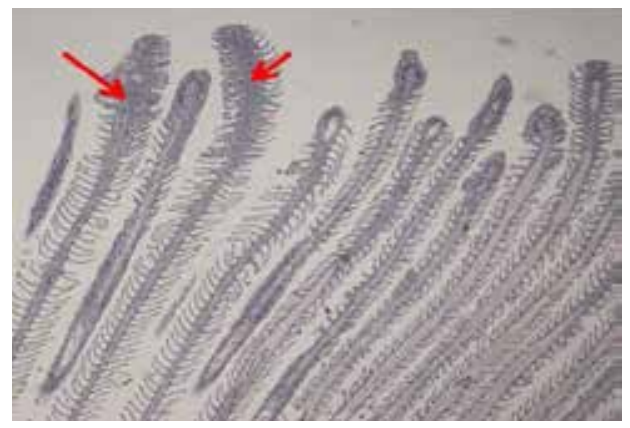

donde se pudo observar necrosis del epiletio lamelar, hiperplasia e hipertrofia lamelar pero fusión lamelar (flecha indicada), fue el mayor daño observado. Se observó además, presencia del parásito Trichodina sp. en las branquias (derecha).

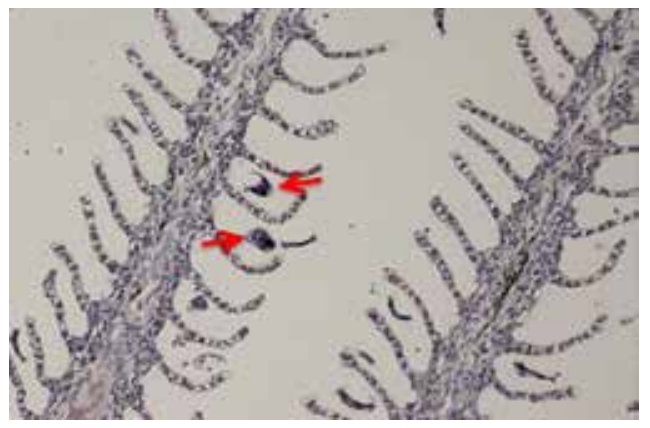

Figura 2. Daños en branquias (4X) y presencia de parásito (20X).

Nota: Laboratorio Continental del IMARPE, Puno (2013). 


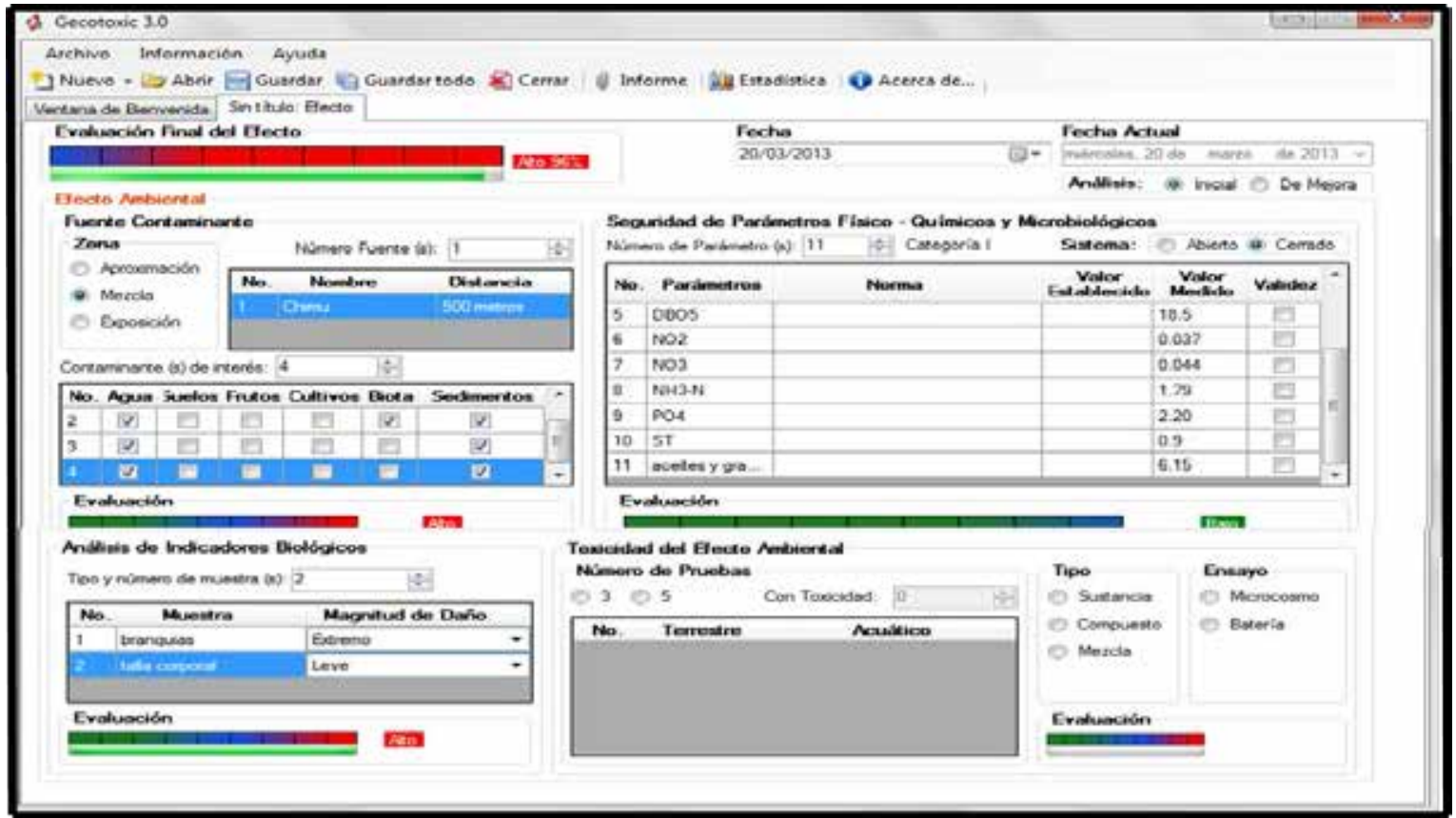

Figura 3. Predicción de riesgo ambiental por GECOTOXIC.

Discusión

En este caso, estudio sobre la mortandad se reporta que el número de ejemplares perdidos fue significativo (119.400), correspondiendo a una biomasa aproximada de 2340.0. La especie con mayor pérdida fue Orestias agassii "carachi gris" y la menor correspondió a Trichomycterus dispar "mauri", existiendo, finalmente, diferencias estadísticamente significativas entre las cuatro especies.

Entre la especie Orestias agassii "carachi gris" y Orestias luteus "carachi amarillo", los individuos observados con mortandad presentaron similar rango de talla media, así como entre Odontesthes bonariensis "pejerrey" y Trichomycterus dispar "mauri".

Dado los resultados anteriores, puede indicarse que el efecto de mortandad ocurrido fue de tal magnitud, que afectó a un gran número poblacional. Resultaron mayormente afectados aquellos ejemplares con tamaño promedio según el intervalo de rango señalado y que correspondió a dos de los principales géneros representados según Lauzanne (1992) como son Orestias y Trichomycterus.
En consideración, las condiciones ambientales tanto de factores bióticos como abióticos sobre los ecosistemas bien definidos pueden ocasionar, sin duda alguna, la aparición de enfermedades en las poblaciones como parte del equilibrio ecológico.

Según Office International des Epizooties (2000), las enfermedades en organismos acuáticos se dividen en "Enfermedades Certificables", siendo aquellas de las que, actualmente, no se dispone de tratamiento alguno para su control y las "Enfermedades Notificables", en las cuales los patógenos causales de enfermedad son susceptibles de ser controlados mediante la aplicación de algún medicamento o sustancia química para su tratamiento, aunque son causantes de grandes mortalidades y finalmente, las "Enfermedades Comunes" que son muy frecuentes y pueden o no causar problemas. El estado de enfermedad se traduce en los peces por la aparición de anomalías del comportamiento: síntomas y/o de la integridad corporal.

Algunas manifestaciones mórbidas son debidas a causas de orden físico, químico o 
biológico de actuación propia o asociada con el fin de perturbar la fisiología animal.

Soares et al. (2011) como Leung \& Bates (2013) mencionan que mundialmente está reconocido entre los mayores impedimentos que frenan el desarrollo de la acuicultura, las enfermedades infecciosas que son un freno para una producción sostenida. En este estudio, pudo observarse secreciones blanco-amarillentas a nivel de los opérculos branquiales de forma abundante, que podrían indicar que algún agente del medio provocó tal aparición. Dichas secreciones, generalmente, se generan cuando ocurren daños agudos en el tejido branquial, que pueden corroborarse con los estudios histopatológicos.

Desde el punto de vista ecotoxicológico, el nivel de estas secreciones puede ser ocasionado por agentes químicos de naturaleza orgánica y bien; quizás como fue el caso por la presencia de agentes patógenos, en que fue diagnosticado, posteriormente, en las branquias de los ejemplares, el parásito Trichodina sp.

De todos los parámetros físico-químicos determinados, la temperatura del agua, oxígeno disuelto y dióxido de carbono, resultaron no solo con valores significativos, sino que al ser parámetros inversamente proporcionales de dependencia se comportaron diferentes a los registros históricos, y fueron corroborados los mismos en la práctica, su interpretación de análisis teórico. De forma similar, ocurrió con las variables meteorológicas, temperatura del aire, velocidad de los vientos y radiación solar.

Fue significativo que días posteriores al evento se intentó tomar muestras de peces en la zona para realizar otros estudios corroborativos donde no pudo capturarse individuos de ninguna especie, y ello indicó entonces que la reaparición tendría una probabilidad impre- decible debido a un posible efecto de repelencia indirecto ecotóxico, cuya fase de latencia o meseta pudiera ser prolongada, dadas las condiciones ambientales del área con niveles de cargas contaminantes (Figura 4).

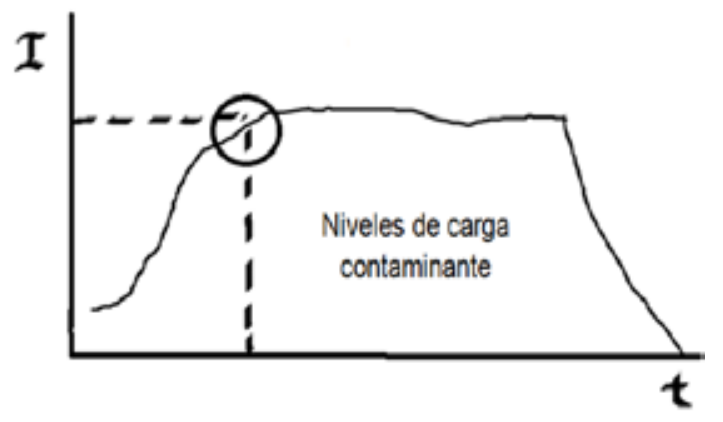

Figura 4. Efecto indirecto de repelencia ecotóxico.

Además, se señala que la interpretación sobre la mortandad de peces fue iniciada con un comportamiento en el aumento de la radiación solar, lo cual incrementó la temperatura del aire y dada la velocidad de los vientos, este influyó sobre la masa de agua de la bahía interior del lago Titicaca.

Sin embargo, debido a que el área de la bahía interior, próxima a la zona de Chimú, está perturbada por las descargas desbordantes de la laguna de oxidación y que, además, es la zona menos profunda hace que la misma, pueda ser una de las más contaminadas, en que las variables meteorológicas al influir sobre ella, determine su alta vulnerabilidad, pudiendo provocar efecto de remoción ecosistémica de tipo local, no reportado con anterioridad para el lago Titicaca. Todo ello pudo incidir luego sobre los parámetros de calidad físico-químicos de agua, haciendo que ocurriera de forma simultánea, interacción por sinergia, adición o potenciación que propició la proliferación ectoparásita con órgano diana en las branquias.

Con todos los resultados obtenidos, los mismos se introdujeron en el programa GECOTOXIC para predecir el riesgo ambiental y que arrojó como resultado ser de tipo alto. 
La predicción de riesgo estimada por GECOTOXIC, está ajustada y presenta similitudes en la comunicación de indicadores referidos por la Environmental Agency Protection (1997), European Communities (2003), Corporación Nacional del Cobre de Chile (2006) y la International Organization for Standardization (ISO, 2009).

De igual modo, el riesgo ambiental comunicado por el programa GECOTOXIC, fue comparado con los resultados estimados en comparación con modelos biológicos en condiciones naturales, cuyos resultados fueron similares (Argota \& Iannacone, 2014b), así como en la predicción de riesgo ecotoxicológico para el río $\mathrm{Ra}$ mis, uno de los tributarios que descargan más contaminantes en el lago Titicaca (Argota et al 2014), por lo que puede indicarse que la predicción de GECOTOXIC para este estudio, tuvo una probabilidad elevada de seguridad.

\section{Referencias}

Argota, P.G. \& Iannacone, O.J. (2014 a). Metodología programada GECOTOX para la predicción de riesgo ecotoxicológico por efluentes y efectos contaminantes en ecosistemas acuáticos. The Biologist, 12(2), 181-193.

Argota, P.G. \& Iannacone, O.J. (2014 b). Similitud en la predicción de riesgo ecológico entre el Software Gecotox y Biomarcadores en Gambusia punctata (Poeciliidae). The Biologist, 12(1), 8598.

Argota, P.G., Miranda P.E. \& Argota, C.H. (2014). Predicción ecotoxicológica de parámetros físico-quimicos, plomo $y$ cadmio en el río Ramis-Cuenca Hidrográfica Titicaca, Puno-Perú. Revista de Investigaciones (Escuela de Posgrado), 5(3), 24-35.

Carpenter, R.A. (1995). Risk assessment. Impact Assessment, 13, 153-187.

Corporación Nacional del Cobre de Chile: (2006). Procedimientos para identificar aspectos ambientales y evaluar el riesgo de sus impactos (Informe). Chile: Autor.

Dambacher, J.M. \& Ramos, J.R. (2007). Understanding and predicting effects of modified interactions through a qualitative analysis of community structure. The Quartely Review of Biology, 82, 227-250.

Darbra, R.M., Eljarrat, E. \& Barceló, D. (2008). How to measure uncertainties in environmental risk assessment. Trends in Analytical Chemistry, 27, 377-385.

Der Oost, R.V., Beyer, J. \& Verneulen, N.P.E (2003). Fish bioaccumulation and biomakers in enviromental risk assessment: a review. Environmental Toxicology and Pharmacology, 13, 57149.

Office International des Epizooties (2000). Diagnostic manual for aquatic animal diseases. París, France: Autor.

Environmental Agency Protection (1997). Ecological risk assessment guidance for superfund. USA: Autor.

European Communities (2003). Technical Guidance Documention Risk Assessment (Informe No. 2). Recuperado del sitio de Internet Joint Research Centre: http://publications. jrc.ec.europa.eu/repository/bitstream/111111111/5619/2/EUR\%20 20418\%20EN-2.pdf

Evans, J.S. (agosto, 2002). Introduction to risk analysis (Slides). Presentado en 
Second course on air quality management (MIT-CAM).

Instituto del Mar del Perú (2013). Mortandad de peces en la bahia interior de Puno del Lago Titicaca. Manuscrito inédito.

International Organization for Standardization (2009). ISO 31000. Risk management - Principles and guidelines. Suiza: Autor.

Lee, S.C. (2009). Environmental risk assessment guidance manual for industrial chemicals. Australia: Environment Protection and Heritage Council.

Leung, T.L.F. \& Bates, A.E. (2013). More rapid and severe disease outbreaks for aquaculture at the tropics: implications for food security. Journal of Aplied Ecology, 50, 215-222.

Management Programme for the Gordon Development (2004). Risk assessment process (Informe No. 9). Recuperado del sitio de Internet Chevron Australia: https://www.chevronaustralia.com

Morgan, M. \& Henrion, M. (1990). Uncertainty: A guide to dealing with uncertainty in quantitative risk and policy analysis. Inglaterra, Cambridge: University Press.
Programa de las Naciones Unidas para el Desarrollo (2003). CEDE-FOA-PELT. La Paz.

Samantray, P., Mishra, B.K., Panda, C.R. \& Rout, S.P. (2009). Assessment of water quality index in Mahanadi and Atharabanki rivers and Taldanda canal in Paradip Area, India. Journal of Human Ecology, 26, 153-161.

Soares, Green, D.M., Turnbull, J.F., Crumlish, M. \& Murray, A.G. (2011). A baseline method for benchmarking mortality losses in Atlantic salmon (Salmo salar) production. Aquaculture, 314, 7-12.

Vanrolleghem P., Young, A., Morris, G., Gandolfi, C. \& Feijtel, T.C. (2001). GREAT-ER: a new tool for management and risk assessment of chemicals in river basins. Contribution to GREAT-ER \#10. Wáter Science and Technology, 43, 179-185.

World Bank (1998). Comparative risk assessment. Recuperado del sitio de Internet del Instituto de Hidrología, Meteorología y Estudios Ambientales: http:// documentacion.ideam.gov.co/openbiblio/bvirtual/001083/Course2/Lecturas/handbook98/comp_risk_assess. pdf 
